\title{
Application and Research of Color in the Facade Design of Elementary School Buildings
}

\author{
Chao Huang \\ Nankai University Binhai College \\ Tianjin, China 300270
}

\begin{abstract}
Color is the most important information symbol for human visual stimulation. It has a very important impact on architecture, especially in primary elementary school buildings. The color environment of the elementary school building refers to the main place and space for pupils to grow up and accept education. It is also the largest "teaching aid" for elementary schools. However, the facade color of the elementary school building can convey a lot of information to the pupils, and affect their psychological changes. In this study, on-site survey and analysis of the facade color of Tianjin elementary school buildings were conducted to find out the problems existing in the facade color design of the elementary school buildings, and then propose the design principles and methods for solving these problems. This research could supplement the theoretical points of facade color design of elementary school buildings in China, thus gradually improving the relatively lagged-behind facade color design of elementary school buildings in China.
\end{abstract}

Keywords-elementary school buildings; architectural color; building facade

\section{INTRODUCTION}

The research on architectural color in China is relatively late. But it has achieved certain results. In 1992, the Institute of Physics of China Academy of Building Research carried out the topic study of "Research on Architectural Color System and Color Card". Based on the study of various quantitative representation methods of colors in the world, relevant experts found a quantitative color description method suitable for the application of Chinese architectural circles. "Research on Architectural Color System and Color Card" has determined China's architectural color system and has taken a gratifying step in promoting the standardization of architectural color design. At the same time, the Institute of Physics of the China Academy of Building Research, the State Administration of Cultural Heritage, the Palace Museum and many other departments participated in the scientific quantitative research on the color standards of classical Chinese architecture. The study on this topic solves the methodological problem in the study of Chinese classical architectural color standards, and proposes a set of typical color standard boards representing the final research results, thereby providing a reliable scientific basis for ancient building maintenance and restoration of China.

The architectural color research and design of foreign countries is gradually developing towards standard and professional. Research topics for architectural colors include local and traditional colors, urban colors, category architectural colors, and individual architectural colors. Depending on the content of the study, the research methods used are different. The study of urban color mainly summarizes the visual effects and design rules of urban color from the perspective of history and aesthetics. This research has been widely recognized in all countries, and its research methods mainly adopt combined research and theoretical analysis methods. Japan began its work in this field. Its research findings have been applied in related fields, and a series of regional color control regulations have been established. During recent years, a color planning center has been established to undertake the research and practice of urban color and related science, thus making the research work more systematic and standardized.

\section{RELEVANT THEORY ON ARCHITECTURAL COLOR SCIENCE}

\section{A. Basic Theory of Color Science}

Color not only has the function of decorating environment, but also conveys environmental information, participates in the formation of environmental style, and affects the user's comfort and aesthetics from the physiological to the psychological aspects. The basic characteristics of color are the three elements of hue, brightness and purification. The three elements can be related, coordinated and applied to create a color system that is aesthetically pleasing and meets environmental needs. [1]

Colors in nature can be divided into two categories achromatic and colored. Achromatic refers to black, white, and gray (different scales of gray); colored refers to all other colors The representation of colors is complex, but the color has three characteristic values, namely: hue, purification, and brightness. These three characteristic values determine that the specific state of color, which is called the "three attributes of colors". Meanwhile, what we see in life is also complexity of three attributes of colors. Therefore, these three attributes are important in the process of color study.

Yu Kongjian, Li Dihua. Sustainable Landscape [J]: Urban Environment, 2007(1) 


\section{B. Architectural Color}

1) Physical function: Color is a feeling of human on the retina when color and light stimulate the human eye. The expression of color cannot be realized without the participation of light, which is determined by the physical properties of color. All objects can reflect light. And the reflectivity varies with the brightness of the surface color of the object. The higher the brightness, the higher the reflectivity is, vice versa. [2] Therefore, there is a difference in the amount of heat absorbed by objects. Objects with high brightness absorb less heat. And object with low brightness absorbs more heat. Experiments have shown that in the case of the same mass of objects, if one is black in surface while the other one is white, they could have different temperatures in sunlight. The temperature of the black surface object is 2-3 degrees higher than that of the white surface object. This likes the clothes we wear. Most of the clothes we wear in the winter are dark and the clothes we wear in the summer are mostly in light color. Therefore, the design of architectural colors should also take full account of this physical function of color. For example, in the northern part of China, the winter needs more radiant energy, so it is more suitable to use warm colors and medium-brightness colors in the exterior color design of the building. In the southern part of China, the summer is very hot, so it is suitable to use some high-brightness colors in design.

2) Regional cultural characteristics: In addition to physiological, psychological and physical functions, the regional cultural characteristics, like the environmental, cultural, customs and times, are also represented on buildings, which make the building style and color unique. In China, because of the strict hierarchy in ancient times, traditional Chinese architectural colors have obvious levels of distinction. For example, buildings of ancient Chinese palaces and houses are different in terms of roofs, walls, doors, windows, and stairs. At the same time, architectural colors also play a symbolic role, and some colors have become synonymous with certain regions and cities. For example, the capital of Nigeria is marked by blue. The capital of Sudan is marked by brown. The buildings in Qingdao of China are marked by red tiles and yellow walls. And the buildings in Suzhou are marked by the black tiles and white walls.

\section{SuRVEY AND CASE ANALYSIS ON THE FACADE COLOR OF TIANJIN ELEMENTARY SCHOOL BUILDINGS}

\section{A. Scope and Method of Survey}

Since the buildings in one city have similar characteristics due to the historical culture and geographical conditions of the same region, in this study, the author aimed to study the architectural color of the elementary schools in Hexi District of Tianjin City to unveil certain problems and solve the problems through the color design of buildings.

\footnotetext{
2 Peng Cainian. Color Art: Introduction to Architectural Art. Beijing:
} China Building Industry Press, 2002.
The survey conducted this time was composed of the following steps: 1. Pre-preparation ( (1) research tool preparation, such as architectural color card, camera, etc. (2) data preparation, such as reviewing some relevant background information before the survey); 2. On-site operation (1) conducted the survey on the exterior color of the elementary school building, including whether the architectural color changes under the sunlight. (2) Interviewed and issued questionnaires to the teachers and students of the school; 3 . Post-processing (analyzed the surveyed results and made into relative charts and tables)

\section{B. Survey on the Facade Colors of Tianjin Elementary School Buildings}

The author conducted a survey and summary on the architectural colors of the 10 elementary schools in the area, and made statistics according to the basic color, the decorated color, and the color collocation of the buildings, and the basic information related to the schools in "Table I". 


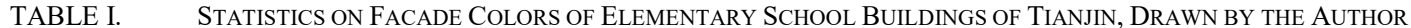

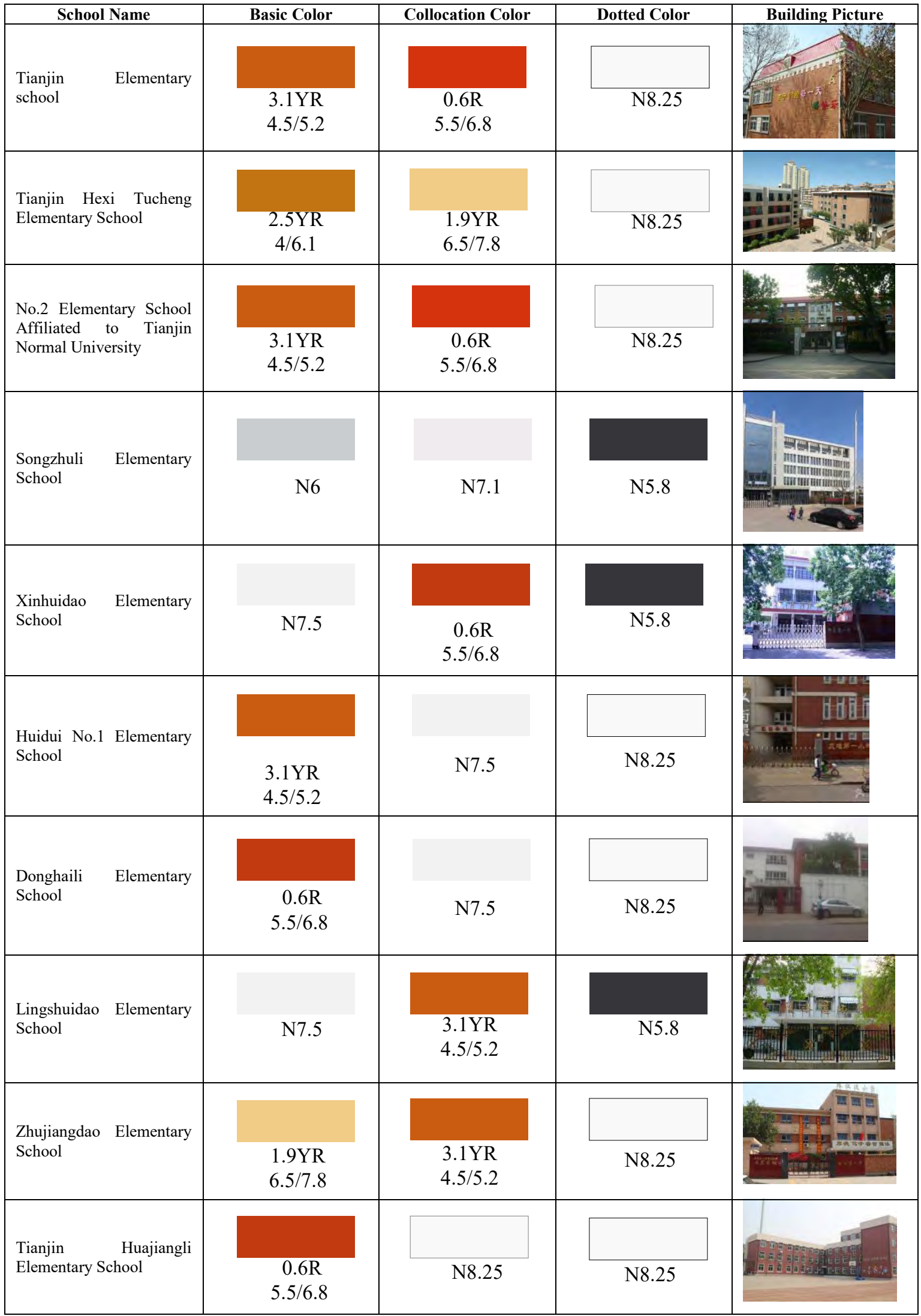


The basic color, collocation color and dotted color in the survey results are defined according to the proportion of a specific color exterior surface of the building. The large proportion color of the outer surface of the building, such as the roof and the wall, is the basic color; the small proportion color on the outer surface of the building, such as the color on the wall, is the collocation color; the color of the small parts such as the door and the window and the small proportion color on the outer surface of the building are dotted color. The representation of the specific color in the table: the achromatic color is represented by $(\mathrm{N})$ plus its brightness value; the colored is represented by its specific hue $(\mathrm{H})$, brightness $(\mathrm{V})$, and purification $(\mathrm{C})$, and marked as $\mathrm{HV} / \mathrm{C}$ (hue brightness/purification). The hue is composed of five essential colors of red (R), yellow (Y), green $(\mathrm{G})$, blue (B), purple (P) and yellow red (YR), and five intermediate colors of green yellow (GY), blue green (BG), purple blue (PB) and red purple (RP) together, to form a basic color. Each basic color is subdivided into 10 parts, which are respectively represented by numbers added to the letters of each color, among which, the No.5 item of each color is the pure color of the basic color.[3]

\section{Facade Color Analysis of Tianjin Elementary School Buildings}

The basic color of the building facades of the elementary schools in Hexi District, Tianjin City, is dominated by yellow red color system, followed by the red and achromatic; and as for the collocation color, it is dominated by achromatic system, followed by red color system and yellow red color system. Therefore, a comprehensive analysis proved that the facade color of the elementary school buildings in Hexi district of Tianjin is mostly of warm color system in "Fig. 1" and "Fig. 2".

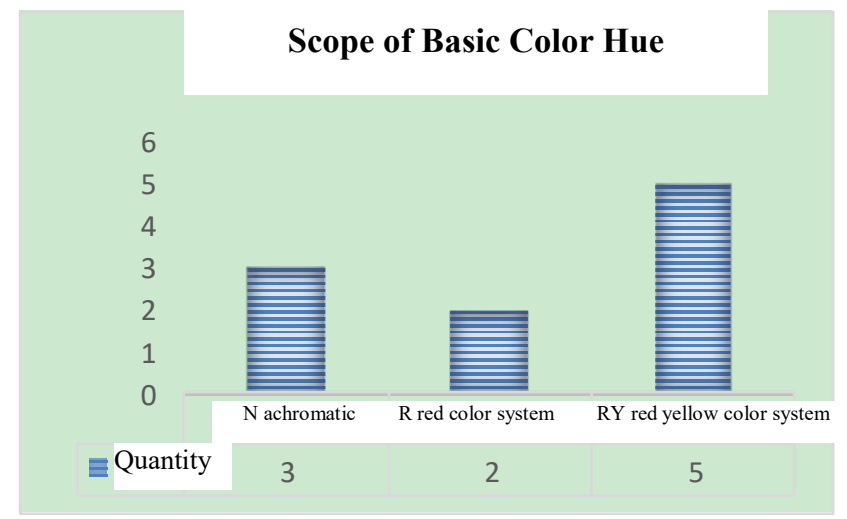

Fig. 1. Statistics on basic color hue of the building facades of Tianjin elementary schools. (drawn by the author)

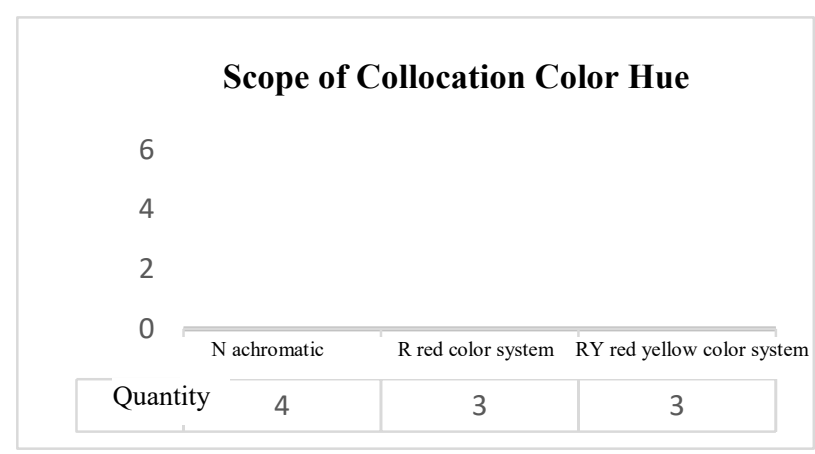

Fig. 2. Statistics on collocation color hue of the building facades of Tianjin elementary schools. (drawn by the author)

The basic facade color of the elementary school buildings is mostly of low brightness. And the brightness distribution of the collocation color is comparatively extensive, with almost half-half proportion of high and low brightness. And the average is relatively average. Therefore, a comprehensive analysis proved that the facade color of the elementary school buildings in Hexi district of Tianjin is featured in stable and balanced attributes. And the color is not too bright or too dark in "Fig. 3" and "Fig. 4".

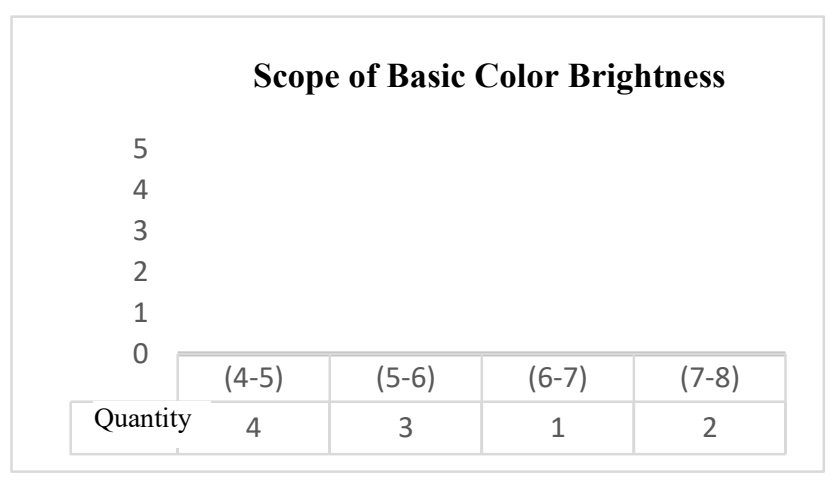

Fig. 3. Statistics on basic color brightness of the building facades of Tianjin elementary schools. (drawn by the author)

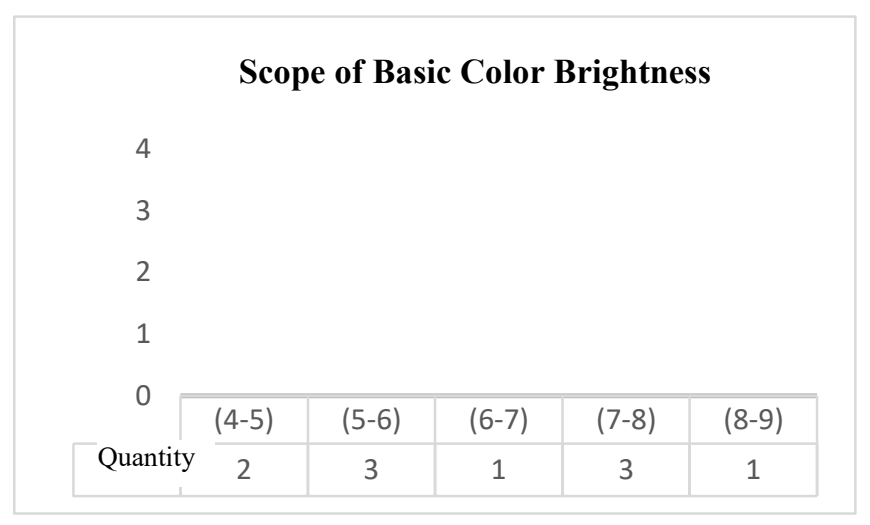

Fig. 4. Statistics on collocation color brightness of the building facades of Tianjin elementary schools. (drawn by the author).

The basic color and the collocation color of the elementary school buildings in Tianjin are all in the range of low to 
medium (7-8) color purification, and most of which are mostly within (7-8). There's few color with high purification. The achromatic color is the largest in quantity in "Fig. 5" and "Fig. 6".

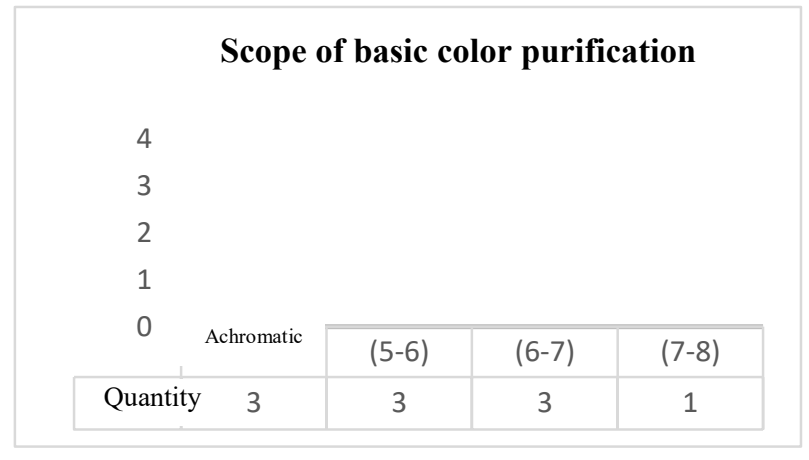

Fig. 5. Statistics on basic color purification of the building facades of Tianjin elementary schools. (drawn by the author)

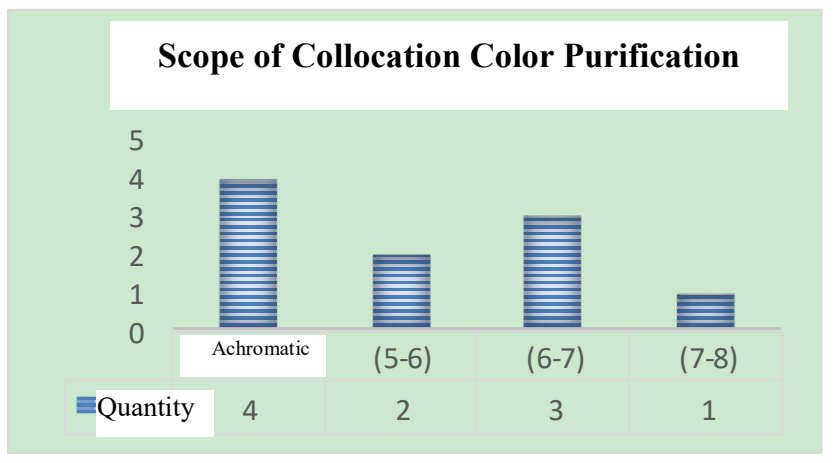

Fig. 6. Statistics on collocation color purification of the building facades of Tianjin elementary schools. (drawn by the author)

\section{Problems Existing in the Facade Color of the Elementary School Buildings in Hexi District}

From the current situation we obtained in this survey and all the above analysis, we can see that there are some problems existing in the facade color of the elementary school buildings in the Hexi District, mainly in the following aspects:

- The color design depth of the facade of the elementary school building is not sufficient. The color design is with general style, not creative, indicating that the design of architectural colors has not attracted much attention. The architectural color design of elementary schools in Western countries is featured in humanized, interesting, and colorful design, which makes the environment good for children growth. It is worth learning.

- The colors of the new and old buildings are not coordinated. In recent years, China has strongly supported education, which has led to the construction of large amount of new teaching buildings or new campuses in many elementary schools. However, the color coordination of new and old buildings has not been considered in the design of architectural colors.
Therefore, the elementary school buildings seem not that much consistent and lack of aesthetics.

- Monotonous materials applied in facade decorating of the elementary school buildings. Most of the finishing materials on the facade of the elementary school buildings are paints and tiles, which greatly limits color and design creativity, lacking of texture nature of the building to some extent.

- There is no regional character in the facade color of the primary school building. The geographical characteristics of color can be the iconic role of the building, making the characteristics of the building more obvious and prominent, and thereby people can identify the building easily.

Through the appearance color investigation for ten elementary schools in Hexi District of Tianjin, and based on the analysis and summarizing, the author further assured the research points: to find out the basic principles and methods for the facade color design of the elementary school building to avoid the problems similar to the above, thereby making the facade color design of the elementary school buildings satisfy the children growth demand better.

\section{DESIGN AND APPLICATION STRATEGY OF THE FACADE COLOR OF THE ELEMENTARY SCHOOL BUILDINGS}

The research is made for being applied in "Wuli Elementary School", located in Wuli Sub-district on the south side of Heiniucheng Road, Hexi District, Tianjin. The school is a medium-to-high-end educational environment with a total construction area of 13,940 square meters. The surrounding areas of the school are mostly commercial and housing buildings, it is of convenient traffic conditions and basically sound infrastructure.

\section{A. Facade Color Design Content of the Elementary School Buildings}

1) Design principle: The facade of the building has certain time and space characteristics no matter viewing from perspective of shape or color. The color of the building facadeis closely related to our daily activities in terms of space or time, and the emotional factors of color play a key role here. Therefore, architectural color will have a great impact on students' learning. Secondly, the architectural color design should also be compatible with the environment. In terms of the facade, it should be coordinated with the surrounding environment, and its background nature should be considered as well. That's because the existence of every architectural image is inseparable from the background relationship. Furthermore, the color of the facade of the building should be acceptable to most people. In particular, the color of the elementary school building must be accepted and understood by the teachers and students to achieve the unity of personality and commonality. At the same time, the color design should also reflect the function and the characteristics of the building. Therefore, the color design of 
the facade of the elementary school building should meet the following principles:

a) The principle of health. In the process of facade color design for the elementary school buildings, we must consider the influence of color on students. And this influence must be positive and healthy, bringing students with a plain, fresh and simple visual enjoyment to promote the healthy growth of students.

b) The principle of fun: Unlike the adults, pupils can be easily attracted by rich and varied colors, so it needs to adopt reasonable and interesting architectural color design to prevent pupils from being influenced by their varying mentality in aspects of learning and growth, while on the other hand, protecting their imagination and curiosity.

c) The principle of particularity: The color design of the facade of the building must reflect its function and personality. It shall make people aware of its function by seeing its appearance and facade color. So the monotonous style is obvious not workable here and it must adhere to the principle of particularity in color design for the building.

2) Design method: The main content of the facade color design of the elementary school building is mainly the color selection. There are two ways to choose colors for the building facade: one is to use color theory, and the other is to use color impression system.

a) Using color theory to select colors: Most buildings are applied with two or more colors to achieve a coordinated effect. And the contrast between the hues includes: the same color contrast, similar color contrast, adjacent color contrast, contrast color contrast and complementary color contrast.

A. Same color contrast. This is the contrast method for the most adjacent colors in hue contrast, and their angle on the hue circle is less than 10 degrees. This color matching method formed by a single hue is kind of safe way with high image uniformity and can express the warm visual effect. Therefore, in the color design of the facade of the elementary school building, the purity and brightness between the colors should be separated by a certain distance to avoid monotony of the image composition.

B. Adjacent color contrast. This is a method for contrast between two neighbouring sets of colors. There is both certain commonality and differences between adjacent colors. Therefore, this color matching method can make the picture more vivid and active in a unified and stable state. It is a harmony beauty for human vision, so it is ideal for color design of facades in elementary school buildings.

C. Contrast color contrast. The contrasting colors refer to colors having 120-150 degree hue angle on the hue circles. The contrast hue is not the same color in the color system, and the contrast is not that strong, so this contrast will form a bright and jumping color impression.

D. Complementary color contrast. The color matching of the complementary hue uses the color at both ends of the diameter of the hue circle, and the two colors are 180 degrees apart, so this is the most stimulating type of all color contrast modes. b) Using color impression system to select color: The method of adopting color theory as the basis for color selection is in line with the aesthetic requirements of the design of the facade of elementary school buildings, and has a beautiful visual effect. However, when selecting the colors for the facade of the elementary school building, it shall also consider the unique architectural properties and functional requirements of the building. Therefore, the color impression system, which was invented by the famous Japanese color psychologist Kobayashi SHIGENOBU, was developed based on such needs. The color impression system uses the scientific and visual method to establish the bridge between functional demand and color selection by taking color psychology as the basis and directly connecting the psychological needs with their corresponding hue according to the color impression and association of color, thereby making us easier to select colors reasonably according to people's emotional impression of color.

The Nippon Color \& Design Research Institute Inc. once investigated the color impressions of the family, school, and company environment. People are asked to express their impressions and psychological needs on various environmental atmospheres by written form, and then corresponding with the color impression system to obtain relative color areas, in which, the school's ambience color impression should be open and healthy - concentrated in the WS area, safe and vibrant concentrated in the CS area "Fig. 7".[4]

\footnotetext{
4 Wen Tao. Wenfeng Color Design [M]. Beijing. China Youth Publishing. 2008: 51-53.
} 


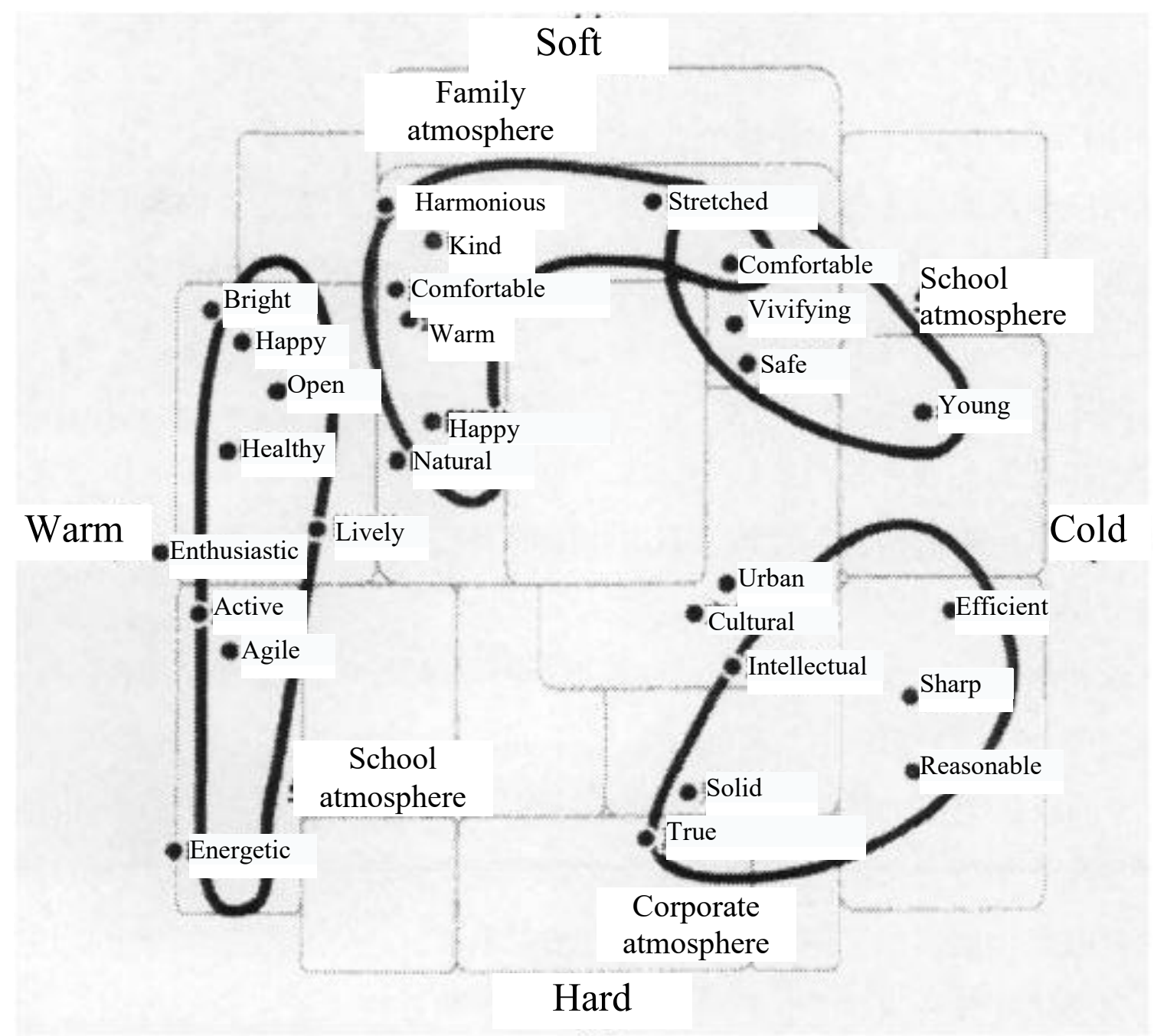

Fig. 7. Different environmental atmospheres and color requirements.

At present, the healthy growth of students has attracted great attention of the public in China. Color is already a basic part of architecture. Good architectural color design can economically and efficiently build a good learning environment, so the color analysis and reasonable color matching are needed in the color design of the facade in elementary school buildings, to improve the quality of the building, and at the same time create an excellent environment for the teachers and students.

\section{CONCLUSION}

The color design of the elementary school building environment hasn't been paid enough attention to in China. This is also a common problem existing in the entire architectural design industry for many years. The author conducted a representative survey on 10 elementary schools in Hexi District, Tianjin, and conducted an in-depth analysis of the facade color of the primary school building, and then summarized the problems existing in the color status, namely the inconsistent color of the new and old buildings, monotonous decorative materials, no characteristics, etc., and designed corresponding design strategies based on the summary of these issues. It is hoped that this research could provide the basis for the facade color design of the elementary school buildings in China in the future, and realize excellent color design and expression in campus architecture in the future, and design a perfect architectural color environment which can bring positive influence to the teachers and students of the elementary schools.

\section{REFERENCES}

[1] Jiao Yan. The dynamic environment color of urban and architectural research [J]. World Architecture, 1998, 5: 57-59. (in Chinese)

[2] (Germany) Eva Heller, translated by Wu Tong. The character of color. Beijing: Central Compilation Press, 2013.1

[3] Jiang Guangxi. Visual performance of color: design color. Tianjin: Tianjin University Press, 2010.5. (in Chinese)

[4] Japanese Architecture Society. Designers talk about architectural color design. Beijing: Electronic Industry Press, 2009.9. 
[5] Zhang Junbo. Analysis on the color system of campus buildings in colleges and universities: [Master's degree dissertation]. Shandong University, 2007. (in Chinese)

[6] Zhang Liangjun. Discussion on the importance of color in architectural design. China New Technology and New Products, 2010, 11: 179. (in Chinese)

[7] Zhang Beibei, Huang Jing. Research on the color design of kindergarten architecture based on children's psychology. Sichuan Architecture, 2009.29(6): 54-55. (in Chinese)

[8] Zucker p. Town and Square from the Agora to the Village Green, New York: Columbia University Press, 1959.

[9] Frank H. Mahnke. Color, Environment, and Human Response[J]. Van Nostrand Reinhold,1996.

[10] Cesar Pelli. Designing with Color[J]. Architectural Design,1996.

[11] Robert Powell, Translated by Weng Hongzhen. Architecture of Learning - New Singapore Schools [M]. Tianjin: Tianjin University Press, 2001.

[12] (UK) Eleanor Curtis, Translated bu Lu Dewei. School Architecture [M]. Dalian: Dalian University of Technology Press, 2004. 